\title{
A Novel Technique in the Use of Fractional Flow Reserve in Coronary Artery Bifurcation Lesions
}

\author{
Justin A. Ratcliffe, M.D. ${ }^{1}$ Yili Huang, D.O. ${ }^{1}$ Tak Kwan, M.D. ${ }^{1}$ \\ ${ }^{1}$ Department of Cardiology, Albert Einstein College of Medicine, \\ Beth Israel Medical Center, New York, New York \\ Address for correspondence and reprint requests Justin A. Ratcliffe, \\ M.D., Department of Cardiology, Albert Einstein College of Medicine, \\ Int J Angiol 2012;21:59-62. \\ Beth Israel Medical Center - Heart Institute, Baird Hall, 5th Floor, \\ First Avenue at East 16th Street, New York, NY 10003 \\ (e-mail: jratcliffe@chpnet.org).
}

\begin{abstract}
Keywords

- coronary artery disease

- interventional cardiology

- fractional flow reserve

- bifurcation lesion

- stent

The side branch (SB) in bifurcation lesions is a frequently encountered challenge that interventional cardiologists must face. There is great interest in determining fractional flow reserve (FFR) in the SB to help guide treatment decisions; however, difficulty with the pressure guidewire limits its widespread use. We propose a novel technique that will ease the incorporation of FFR in routine evaluation of bifurcation lesions, and allow better assessment of provisional stenting and need for SB intervention. Conventionally, to measure SB FFR, the jailed SB is re-crossed through the stent strut using a pressure wire. Our technique involves the use of a microcatheter and wire exchange within the SB, thereby alleviating some of the technical difficulties associated with the FFR wire. In light of the difficulties and clinical concerns associated with bifurcation lesions, we propose an innovative method to ease the incorporation of FFR to allow better assessment of provisional stenting and need for SB intervention.
\end{abstract}

The bifurcation lesion is one of the most challenging lesion subsets in the field of interventional cardiology. Compared with nonbifurcation lesions, bifurcation intervention is associated with more complex procedures, lower procedural success rates, and higher clinical event rates. ${ }^{1,2}$ Options for the operator treating the bifurcation lesions include provisional stenting or a two-stent approach. However, many studies have reported that the two-stent approach, stenting both the main vessel and side branch (SB), did not offer any advantages over stenting of the main vessel only and was associated with procedural difficulty and higher complications. ${ }^{3-6}$ Provisional stenting is the default approach for most bifurcation lesions. To perform final kissing balloon inflation after main vessel stenting is still a controversial subject. Recently, the Nordic-Baltic Bifurcation Study III $^{7}$ showed that main vessel stenting with or without final kissing balloon dilatation was associated with similar clinical outcomes; however, these results cannot yet be applied to all types of bifurcation lesions.

Recently, there is great interest in the determination of fractional flow reserve (FFR) in the SB before intervention.
However, in practice, the FFR pressure guidewire is difficult to control and manipulate in certain SB anatomy, leading to increased fluoroscopy time and contrast dye usage. We propose the use of a workhorse guidewire for easier engagement of the SB lesion with subsequent exchange catheter through the lesion, and then final exchange for the FFR wire. The benefits of FFR can then be applied without the procedural difficulty usually associated with FFR pressure guidewire placement. We will further discuss this innovative technique and hope to ease some of the difficulty associated with treating coronary artery bifurcation lesions.

\section{Methods}

Bifurcation percutaneous coronary intervention (PCI) is performed using the provisional stenting approach with a 5F- or $6 \mathrm{~F}$-coronaryguiding catheter. After inserting guidewires into the main branch and SB, the SB guidewire is jailed after a stent is implanted in the main branch ( $\mathbf{- F i g . 1}$ ). Conventionally, to measure SB FFR, the jailed SB is re-crossed through the stent strut using a pressure wire. Due to the technical difficulties of
Copyright @ 2012 by Thieme Medical Publishers, Inc., 333 Seventh Avenue, New York, NY 10001, USA. Tel: +1(212) 584-4662.
DOI http://dx.doi.org/ 10.1055/s-0032-1306419. ISSN 1061-1711. 

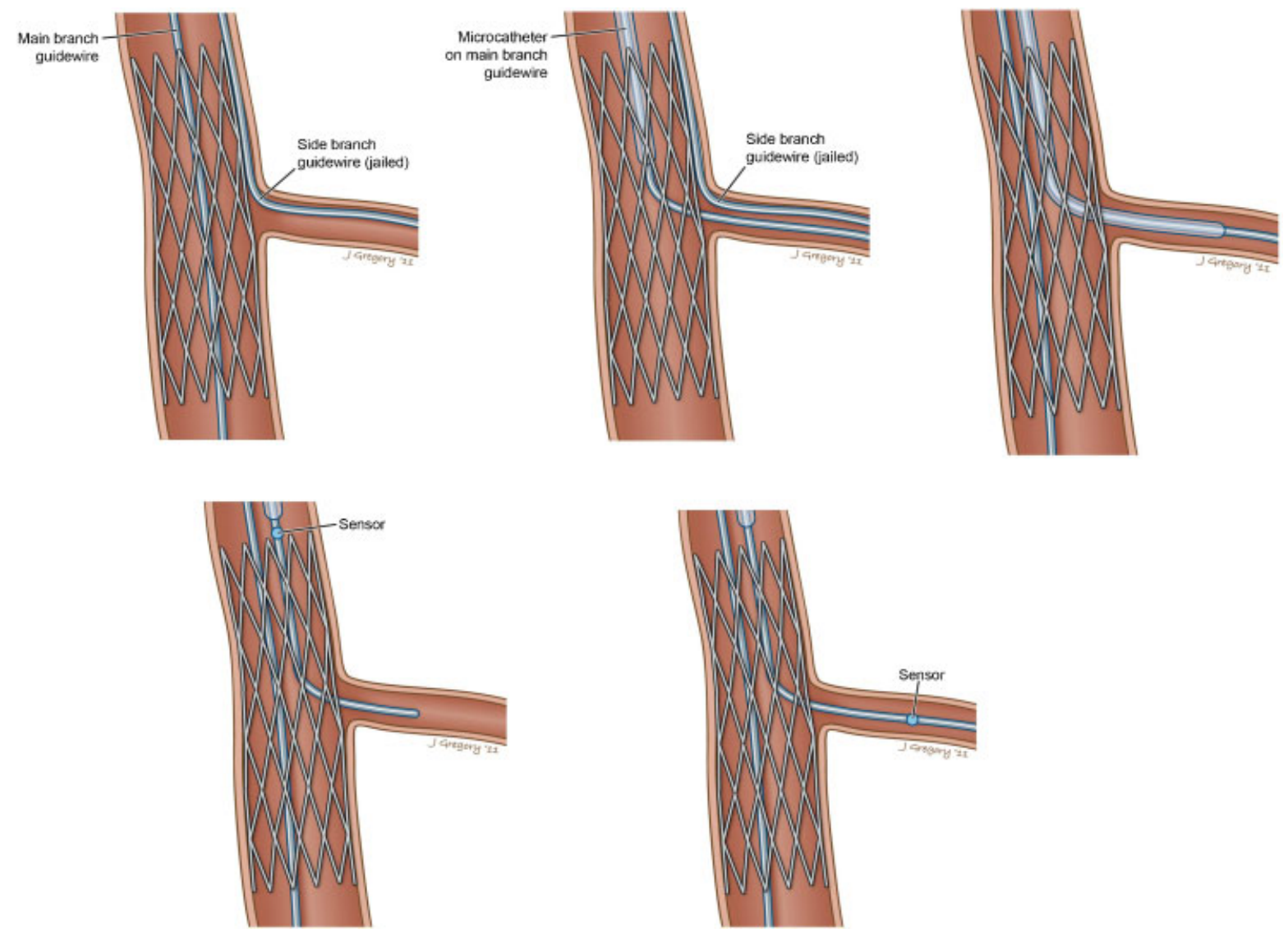

Figure 1 Fractional flow reserve (FFR) use in coronary artery bifurcation lesions: Top, Left: Routine placement of a main branch guidewire and jailed side branch (SB) guidewire after stent implantation. Top, Middle: The main wire is loaded into a microcatheter and inserted into the SB with guidance from the jailed wire. Top, Right: The originally jailed SB wire is then withdrawn and inserted into the main branch. Bottom, Left: The pressure wire can then be inserted into the microcatheter and advanced into the SB. After the microcatheter is withdrawn into the guiding catheter, the pressure wire can slowly be withdrawn proximally to the SB ostial lesion to normalize the pressure wire. Bottom, Right: The pressure wire is then advanced past the SB ostial lesion.

the pressure wire, we propose a new method to increase the success of FFR usage. After stent implantation, the main wire is withdrawn and loaded into a microcatheter. A workhorse guidewire is inserted into the $S B$ with the guidance of the jailed wire in the SB. The originally jailed SB wire is then withdrawn and inserted into the main branch. The pressure wire can then be inserted into the microcatheter and advanced into the SB. The microcatheter is withdrawn into the guiding catheter while keeping the pressure wire in the SB. The pressure wire is slowly withdrawn proximally to the SB ostial lesion. Normalization of the pressure is performed proximally to the SB ostium and then advanced past the SB ostial lesion. This provides the real FFR of the SB ostial lesion provided a proximal lesion is absent. (Of course, when stenting the main branch lesion, a proximal lesion should be absent.) However, if a proximal lesion within the main branch is present, then the pressure wire can be normalized inside the guiding catheter before reinserting into the microcatheter. Once an accurate FFR is obtained, the operators can then make an informed decision regarding the treatment of the SB lesion.

\section{Results}

This technique was successfully performed in 10 patients with difficult SB anatomy. The FFR wire was smoothly transitioned into the SB and measured in these patients, without procedural difficulty in $100 \%$ of the cases. In another 10 patients with bifurcation lesions, FFR was measured in the SB using the conventional technique. However, there were three patients in this group who required crossover to the new, proposed technique after prolonged and unsuccessful attempts at SB guidewire insertion. In comparison to the conventional technique, the new technique had a higher success rate and shorter procedural time.

\section{Discussion}

FFR is a widely used technique in cardiac catheterization laboratories to determine the functional significance of coronary artery stenosis. It measures the ratio of maximal blood flow achievable in a stenotic vessel compared with the theoretical maximal flow in the same vessel if no stenosis was present. Stenosis with an FFR $<0.75$ is almost invariably 
associated with inducible myocardial ischemia. FFR has certain distinct advantages and is different than other modalities, such as coronary angiography or intravascular ultrasound, to evaluate coronary stenosis. Generally, FFR takes into account size of the myocardium, significance of an obstructive lesion, and translates it into the amount of myocardial ischemia in the particular lesion. The FAME ${ }^{8}$ trial revealed that FFR-guided PCI resulted in superior clinical outcomes than using angiographic guidance alone.

Determination of significance of the SB lesion is a difficult task. Angiographic evaluation for these lesions is more challenging than for usual stenotic lesions and intravascular ultrasound is difficult to perform in these cases unless the SB ostium is dilated. Koo et $\mathrm{al}^{9}$ showed that qualitative coronary analysis, as compared with FFR, generally overestimated the functional severity of stenosis in the assessment of jailed SB lesions likely secondary to the eccentricity of the lesion, stent strut artifacts, or a relatively small SB myocardial territory. As a follow-up study, Koo et al ${ }^{10}$ compared functional outcomes in patients with bifurcation lesions whose treatment was guided with and without FFR in the SB lesion. In comparison to patients with similar bifurcation lesions but without FFR guidance, the FFR-guided subset had similar 9-month cardiac event rates. Additionally, the percentage of lesions receiving further intervention was higher in the non-FFR-guided group. However, the lack of randomization and small study size limit the findings of this study, but does support the concept that angiographic evaluation alone may overestimate the severity of SB lesions resulting in unnecessary intervention. Another study ${ }^{11}$ investigating the mechanism of SB stenosis again demonstrated the difficulty with anatomical evaluation. SB lumen area can be underestimated due to individual variability and complex mechanisms including the extent of plaque shift and carina shift, amount and location of lesions, and bifurcation angles.

FFR has the ability to help in the functional assessment of the SB in bifurcation lesions and facilitate treatment decisions. However, the FFR wire has notoriously known to be a very flimsy coronary wire with difficult torquability. Many times due to significant ostial branch lesions with different orientation and takeoffs and after provisional stent placement, insertion of FFR becomes very difficult and cumbersome, which requires increased fluoroscopy time and dye usage. Nowadays, there are many different workhorse guidewires preferred by different operators. These workhorse guidewires can engage SB lesions both through stents and ostial lesions easily due to its great support, increased torquability, sterriability, lubricity, and tactile feedback. The low traumacity, and easiability has made it a great wire to engage these SB lesions during postprovisional stenting. Our proposed technique is to initially use workhorse guidewires to engage SB lesions, and subsequently place exchange microcatheter through lesion. Then withdraw the guidewire and easily exchange for FFR wire. To ease the need to calibrate the wire, we would withdraw the proximal end of the wire sensor proximally of the SB ostium. This innovative method can ease the technical difficulty associated with FFR wire usage and therefore increase procedural success rates. Not only will the operator obtain a better assessment of provisional stenting and need for SB intervention, but operation time, fluoroscopy time, and contrast volume should also be decreased by this novel technique.

The use of FFR is very important for the physiologic evaluation of bifurcation lesions. FFR-guided interventions are feasible and can prevent unnecessary complex coronary interventions and its related complications. We propose that this new method will facilitate timely, clinically, and economically sound decisions in treating bifurcation lesions.

\section{Limitations}

This technique may add the extra cost of an additional guidewire and microcatheter. Also, the pull back of the proximal end of the pressure wire to the main vessel is accurate provided that there is no obstructive lesion in the proximal main vessel. Otherwise, the pressure wire can be normalized inside the guiding catheter before reinserting into the microcatheter, which is not the ideal FFR measurement.

\section{Conclusion}

In light of the difficulties and clinical concerns associated with bifurcation lesions, we propose an innovative method to ease the incorporation of FFR to allow better assessment of provisional stenting and need for SB intervention.

\section{References}

1 Iakovou I, Ge L, Colombo A. Contemporary stent treatment of coronary bifurcations. J Am Coll Cardiol 2005;46(8):1446-1455

2 Hildick-Smith D, de Belder AJ, Cooter N, et al. Randomized trial of simple versus complex drug-eluting stenting for bifurcation lesions: the British Bifurcation Coronary Study: old, new, and evolving strategies. Circulation 2010;121(10):1235-1243

3 Steigen TK, Maeng M, Wiseth R, et al; Nordic PCI Study Group. Randomized study on simple versus complex stenting of coronary artery bifurcation lesions: the Nordic bifurcation study. Circulation 2006;114(18):1955-1961

4 Ferenc M, Gick M, Kienzle R-P, et al. Randomized trial on routine vs. provisional T-stenting in the treatment of de novo coronary bifurcation lesions. Eur Heart J 2008;29(23):2859-2867

5 Colombo A, Bramucci E, Sacca S, et al. Randomized study of the crush technique versus provisional side-branch stenting in true coronary bifurcations: the CACTUS (Coronary Bifurcations: Application of the Crushing Technique Using Sirolimus-Eluting Stents) Study. Circulation 2009;119:71-78

6 Korn HV, Yu J, Ohlow MA, et al. Interventional therapy of bifurcation lesions: a TIMI flow-guided concept to treat side branches in bifurcation lesions-a prospective randomized clinical study (Thueringer bifurcation study, THUEBIS study as pilot trial). Circ Cardiovasc Interv 2009;2(6):535-542

7 Niemelä M, Kervinen K, Erglis A, et al; Nordic-Baltic PCI Study Group. Randomized comparison of final kissing balloon dilatation versus no final kissing balloon dilatation in patients with coronary bifurcation lesions treated with main vessel stenting: the Nordic-Baltic Bifurcation Study III. Circulation 2011;123(1): 79-86 
62 Treatment of Bifurcation Lesions Using FFR Ratcliffe et al.

8 Tonino PA, De Bruyne B, Pijls NH, et al; FAME Study Investigators. Fractional flow reserve versus angiography for guiding percutaneous coronary intervention. N Engl J Med 2009;360(3): $213-224$

9 Koo BK, Kang HJ, Youn TJ, et al. Physiologic assessment of jailed side branch lesions using fractional flow reserve. J Am Coll Cardiol 2005;46(4):633-637
10 Koo BK, Park KW, Kang HJ, et al. Physiological evaluation of the provisional side-branch intervention strategy for bifurcation lesions using fractional flow reserve. Eur Heart J 2008;29(6): 726-732

11 Koo BK, Waseda K, Kang HJ, et al. Anatomic and functional evaluation of bifurcation lesions undergoing percutaneous coronary intervention. Circ Cardiovasc Interv 2010;3(2):113-119 